\title{
X-ray imaging of dispersive charge modes in a doped Mott insulator near the antiferromagnet/ superconductor transition
}

\author{
Y. W. Li, ${ }^{1}$ D. Qian, ${ }^{1}$ L. Wray, ${ }^{1}$ D. Hsieh,,${ }^{1}$ Y. Xia,${ }^{1}$ Y. Kaga, ${ }^{2}$ T. Sasagawa, ${ }^{2}$ H. Takagi, ${ }^{2}$ R. S. Markiewicz,,${ }^{3}$ A. Bansil,${ }^{3}$ \\ H. Eisaki, ${ }^{4}$ S. Uchida, ${ }^{2}$ and M. Z. Hasan ${ }^{1}$ \\ ${ }^{1}$ Department of Physics, Joseph Henry Laboratories of Physics, Princeton University, Princeton, New Jersey 08544, USA \\ ${ }^{2}$ Department of Physics, University of Tokyo, Tokyo 113-8656, Japan \\ ${ }^{3}$ Department of Physics, Northeastern University, Boston, Massachusetts 02115, USA \\ ${ }^{4}$ AIST, 1-1-1 Central 2, Umezono, Tsukuba, Ibaraki 305-8568, Japan
}

(Received 8 January 2008; published 15 August 2008)

\begin{abstract}
Momentum-resolved inelastic resonant x-ray scattering is used to map the doping evolution of bulk electronic modes in the doped Mott insulator class $\mathrm{Nd}_{2-x} \mathrm{Ce}_{x} \mathrm{CuO}_{4}$. As the doping induced antiferromagnet/ superconductor (AFM/SC) transition is approached, we observe an anisotropic redistribution of the spectral weight of collective excitations over a large energy scale along the $\Gamma \rightarrow(\pi, \pi)$ direction, whereas the modes exhibit broadening $(\sim 1 \mathrm{eV})$ with relatively little softening along $\Gamma \rightarrow(\pi, 0)$ with respect to the parent Mott state $(x=0)$. Our study reveals a closing of the charge gap in the vicinity of the zone center even though the mode softening and spectral redistribution involve an unusually large energy scale over the full Brillouin zone. The collective behavior of modes in the vicinity of the AFM/SC critical transition is demonstrated.
\end{abstract}

DOI: 10.1103/PhysRevB.78.073104

The evolution of a strongly correlated material with doping-from a Mott insulator to a conducting metal-is one of the most intensively studied issues in modern condensed matter physics. This fascinating evolution has proven to be full of surprises, such as the appearance of high- $T_{c}$ superconductivity, non-Fermi liquid behavior, and nanoscale phase separation. ${ }^{1}$ Mott insulators often exhibit phase transitions upon doping, which are signaled or hallmarked by the softening or redistribution of the spectral weight of collective charge or spin modes. The behavior of spin modes has been extensively investigated via neutron scattering. ${ }^{2}$ Although charge excitations near the Brillouin zone (BZ) center can be accessed by optical techniques, ${ }^{3}$ their behavior with momentum over the full BZ remains largely unexplored. Here, as demonstrated in recent experimental ${ }^{4,5}$ and theoretical studies, ${ }^{6,7}$ inelastic $\mathrm{x}$-ray scattering provides such a unique opportunity. While previous studies have focused largely on either undoped one-dimensional ${ }^{8}$ or two-dimensional ${ }^{4,5}$ insulators, or hole-doped superconductors, ${ }^{9}$ Mott insulators can be doped with electrons as well. In fact, it appears that with electron doping, cuprate bands evolve in a much more straightforward and systematic manner ${ }^{10-14}$ than with hole doping. A limited previous work ${ }^{15}$ that focused on a superconductor with a fit to the one-band theory does not provide insights into the way the collective modes of a nonsuperconducting Mott insulator evolve into a superconductor wherein the high-energy excitations of the Mott insulator are intimately connected to the lower-energy physics of the superconductor. Here, we report a high-resolution study of the evolution of the collective charge excitations of the Mott insulating state $(x=0)$ with electron doping in approaching the critical antiferromagnet/superconductor (AFM/SC) transition. Our finding, which was made possible by studying the doped insulating states, is that as the electron-doping induced $\mathrm{AFM} / \mathrm{SC}$ transition is approached from the $x=0$ Mott side, the system exhibits anisotropic softening of the excitations over a large energy scale $(\sim 5 \mathrm{eV})$ along the $\Gamma \rightarrow(\pi, \pi)$ direction, whereas the modes exhibit broadening $(\sim 1 \mathrm{eV})$
PACS number(s): 78.70.Ck, 74.20.Mn, 74.25.Jb, 74.72.-h with relatively little softening along $\Gamma \rightarrow(\pi, 0)$. Our results suggest that a multiband Hubbard model is essential to describe the evolution from the $x=0$ Mott state to the superconductor since the high-energy and the low-energy excitations evolve into one another. Moreover, our results show that the evolution of the Mott physics of an electron-doped cuprate is dramatically different from that previously reported in hole-doped cuprates. ${ }^{9}$

The electronic structure of $\mathrm{Nd}_{2-x} \mathrm{Ce}_{x} \mathrm{CuO}_{4}$ (NCCO) has been studied by angle-resolved photoemission (ARPES) and optical spectroscopies. ARPES studies ${ }^{16}$ have found that the electrons directly dope into the bottom of the upper Hubbard band and yield small Fermi surface (FS) pockets, with a crossover around optimal doping to a large FS. Such a scenario, wherein the magnetic order remains commensurate without signs of "stripe" (charge inhomogeneity) or other phase separation, also describes magnetization ${ }^{13,17,18}$ and optical data. ${ }^{19}$

The experiments were performed at CMC-CAT beamline 9-ID-B at the Advanced Photon Source. Resonant inelastic $\mathrm{x}$-ray scattering (RIXS) at the copper $K$ edge allows a large enough momentum transfer to cover several BZs. The scattered photon energy was measured by using a diced Ge (733) crystal analyzer, and the intensity was recorded by a solid state detector. The overall energy resolution was set to about $0.37 \mathrm{eV}$ in order to improve count efficiency, enabling us to detect the fine momentum-dependent details of the spectra. All data were taken at room temperature. To avoid possible polarization induced artifacts when measuring the in-plane anisotropy, the sample was mounted with the incident polarization directed along the $c$ axis [Fig. 1(a)]. The data were collected at several values of the momentum transfer vector $\mathbf{q}=\mathbf{k}_{\mathbf{i}}-\mathbf{k}_{\mathbf{f}}$ in the second $\mathrm{BZ}$ along the [100] direction and in the fourth BZ along the [110] direction. We carefully set the $q_{z}$ component to zero to avoid contamination of unwanted and spurious $d d$ modes that mix with the Mott gap excitations. We first examined the detailed incident energy dependence of the loss spectra at several momenta. Representative 


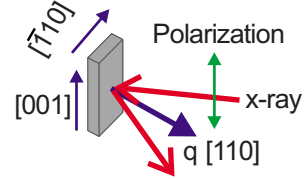

(a)

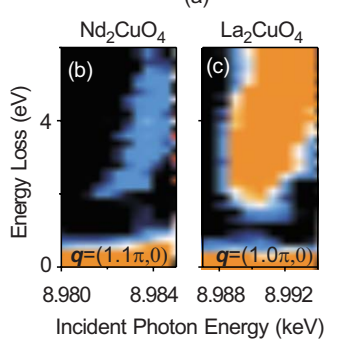

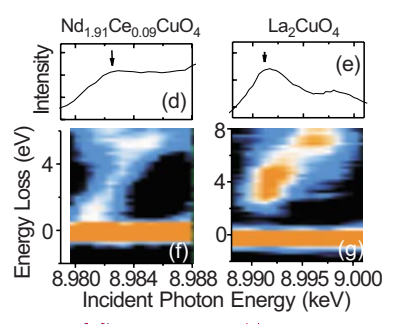

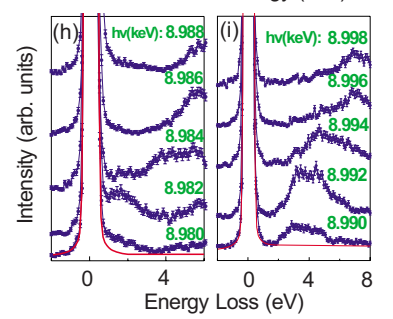

FIG. 1. (Color online) Incident energy dependence of electronic excitations. (a) Vertical scattering geometry employed with an x-ray field along [001]. [(b) and (c)] X-ray energy dependence of inelastic excitations for $\mathrm{Nd}_{2} \mathrm{CuO}_{4}$ and $\mathrm{La}_{2} \mathrm{CuO}_{4}$. The $Q$ values are chosen to minimize the large quasielastic background. [(d)-(g)] Excitations near the Mott gap are seen to be enhanced near the first absorption peak, not only in the undoped insulator but also in the doped system. A few energy-loss curves corresponding to the data images in (f) and (g) are shown in (h) and (i). The red (dark gray) curves show the fits to the quasielastic data.

data sets are presented in Fig. 1. It is known that the chargetransfer gap excitations resonate near the absorption peaks. ${ }^{9}$ A similar resonance behavior is seen in NCCO $x=0$, which is similar to that in the more extensively studied $\mathrm{La}_{2-x} \mathrm{Sr}_{x} \mathrm{CuO}_{4} .{ }^{9}$ However, the scattering intensity at the lowenergy branch is about an order of magnitude weaker. Our systematic investigations that are summarized in Fig. 1 show that a similar shape of the resonance profile is seen for the lower-energy excitations in the electron-doped system. Accordingly, we employed a photon energy of $8.982 \mathrm{keV}$ (this energy is above the $1 s->3 d$ edge that suppresses crystalfield excitations ${ }^{20}$ ).

Figure 2 summarizes our RIXS data on NCCO. In the undoped system in Fig. 2(a), a broad excitation is observed near the zone center around $2 \mathrm{eV}$ with an onset energy of $\approx 1 \mathrm{eV}$, which is consistent with the charge-transfer gap found in this compound in optical studies. ${ }^{21}$ It is seen to lose intensity as it approaches the zone corner along the $[\pi, \pi]$ direction as well as the $[\pi, 0]$ direction [Fig. 2(a)]. Along $[\pi, \pi]$, it merges at the zone corner with a higher excitation band near $5 \mathrm{eV}$ that disperses upward. With doping, these two high-energy branches split at $(\pi, \pi)$, as seen in Figs. 2(b) and 2(c), but only the lower-energy branch significantly softens (i.e., moves to a lower energy), so that the overdoped system in Fig. 2(c) displays a large excitation gap at the $(\pi, \pi)$ point from 2 to $4 \mathrm{eV}$. In sharp contrast, the aforementioned $2 \mathrm{eV}$ branch uniformly evolves along $[\pi, 0]$ and rapidly softens near the zone center as it tends to close the excitation gap with doping. Notably, the zero-loss energy is not accessible due to the presence of the strong quasielastic peak [see, e.g., Fig. 1(h)], making it difficult to extract significant data below $\approx 0.4 \mathrm{eV}$, where the fitting and subtraction of the quasielastic peak leads to uncertainties. Neverthe-

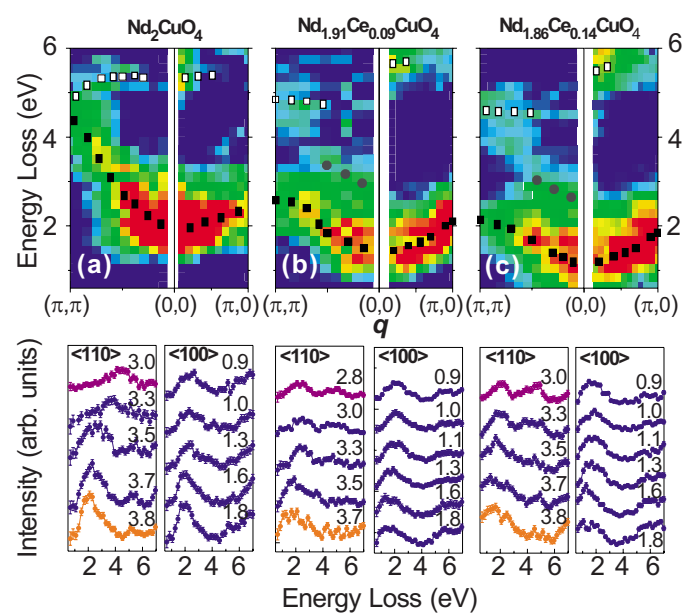

FIG. 2. (Color online) Doping evolution of charge excitations in NCCO: (a) $x=0$ (parent Mott state), (b) $x=0.09$ (doped AFM), and (c) $x=0.14$ (superconductor). The upper row shows intensity maps in the reduced zone by using a color scheme wherein high intensity is denoted as red (dark gray) and low intensity as blue (black). The black, gray, and white dots are guides to the eye for the dispersion of low-, medium-, and high-energy excitation branches, respectively. The lower row gives a few representative spectra corresponding to the images in the upper row. Absolute momentum values (in units of $\pi / a_{o}$ ) are shown attached to various spectra and lie in the fourth BZ along [110] and the second BZ along [100].

less, the softening of the low-energy excitations is evident in the data over the BZ.

The preceding observations are further highlighted through the directly measured data curves presented in Fig. 3. Doping dependent changes in the individual energy-loss curves are compared in Fig. 3(a). Along [110], doping splits the peak in the undoped spectrum around $5 \mathrm{eV}$ at $(\pi, \pi)$ [red uppermost curve on the left side of Fig. 3(a)] into two peaks, and the lower of these peaks rapidly softens with doping. In contrast, along [100] a monotonic softening of the lowenergy excitations is found. However, our work does not rule out a spectral weight transfer at lower energies. The changes in the positions of various spectral features and some of the
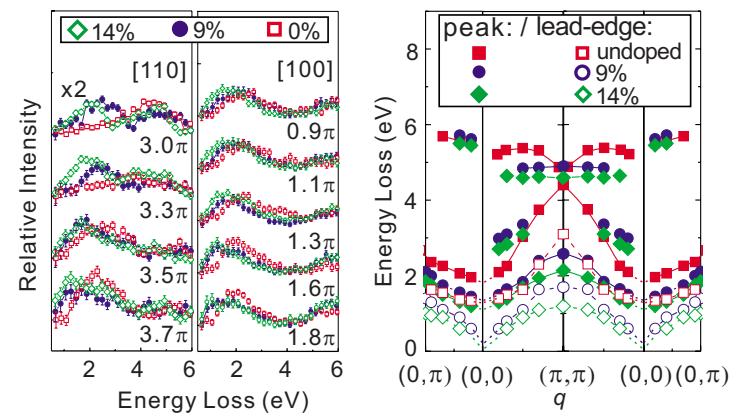

FIG. 3. (Color online) (a) Spectral curves (raw data) along [110] and [100] for the undoped system [red (dark gray) squares] are compared at various momenta to the corresponding spectra for the doped system [black circles and green (gray) diamonds]. (b) The positions of peaks [marked in Figs. 2(a)-2(c)] based on the center of gravity method (Refs. 4 and 8 ) of various spectral features (filled symbols) and some of the associated leading edges (open symbols) as a function of momentum and doping are plotted. 
associated leading edges are plotted in Fig. 3(b) as a function of momentum and doping. The upper branches around 5-6 $\mathrm{eV}$ are seen to be weakly affected by doping. Despite significant softening of one of the modes around $(\pi, \pi)$, the closing of the excitation gap is limited to the region of the zone center as one approaches the superconducting phase $(x$ $=0.14$ ). These results are in clear contrast to the behavior in hole-doped cuprates such as the $\mathrm{La}_{2-x} \mathrm{Sr}_{x} \mathrm{CuO}_{4}$ or the $\mathrm{YBa}_{2} \mathrm{Cu}_{3} \mathrm{O}_{7+d}$ series. $^{9}$

To interpret the present data in terms of RIXS spectra computed within the framework of a three-band Hubbard Hamiltonian of $\mathrm{NCCO}$, based on $\mathrm{Cu} d_{x^{2}-y^{2}}$ and two $\mathrm{O} p_{\sigma}$ orbitals, we extend the theoretical framework described in Ref. 7 by incorporating the doping evolution data made available here. The specific values of the parameters used in this work to fit the spectra are $t_{\mathrm{CuO}}=0.85 \mathrm{eV}, t_{\mathrm{OO}}=$ $-0.6 \mathrm{eV}, \Delta_{0}=-0.3 \mathrm{eV}$, and $U_{p}=5.0 \mathrm{eV}$, where $t_{\mathrm{CuO}}$ and $t_{\mathrm{OO}}$ are the $\mathrm{Cu}-\mathrm{O}$ and $\mathrm{O}-\mathrm{O}$ nearest neighbor hopping parameters, $n_{d}\left(n_{p}\right)$ is the average electron density on $\mathrm{Cu}(\mathrm{O}), m_{d}$ is the average electron magnetization on $\mathrm{Cu}$, and $U\left(U_{p}\right)$ is the $\mathrm{Cu}$ (O) on-site Coulomb repulsion. The remaining parameter, crucial for fitting the doping evolution of the excitations, the Hubbard $U$, is taken to be $7.45 \mathrm{eV}$ at $x=0$ with a weak doping dependence: $U=6.69 \mathrm{eV}$ at $x=0.09$ and $U$ $=6.27 \mathrm{eV}$ at $x=0.14$, so that the effective $U$ decreases by about $16 \%$ over this doping range, presumably reflecting the effects of screening. We note that before proceeding with RIXS computations, we self-consistently determined the chemical potential and the magnetization $m_{d}$ on $\mathrm{Cu}$ sites at each doping level. The $m_{d}$ values so derived were 0.32 at $x$ $=0,0.19$ at $x=0.09$, and 0.12 at $x=0.14$.

Figure 4 (top) shows the calculated RIXS intensity maps. The positions of various experimentally observed spectral peaks (filled circles) and the leading edge of the low-energy feature (filled diamonds) are superposed for ease of comparison. The high-energy peaks involve transitions from the nonbonding $\mathrm{O}$ and bonding $\mathrm{Cu}-\mathrm{O}$ bands to unoccupied states in the antibonding band, and fall in the same energy range as transitions involving other $\mathrm{Cu}$ and $\mathrm{O}$ orbitals that are not included in the present three-band model. Therefore, it is appropriate to concentrate on the behavior of RIXS peaks within a few electron volts, which are associated with the antibonding $\mathrm{Cu}-\mathrm{O}$ band, which is split by antiferromagnetic ordering. In this energy region, the theoretically predicted changes in the energies of various features as a function of momentum and doping are in reasonable accord with experimental observations highlighted in the discussion of Figs. 2 and 3 above. In particular, the softening of low-energy peaks is well reproduced and the closing of the gap occurs near $\Gamma$. The latter effect can be readily understood. The RIXS transitions involve both intraband and interband contributions. In the absence of a gap, only intraband transitions are possible near $\Gamma$, which can only exist near a zero-energy transfer close to the Fermi level. Away from $\Gamma$, intraband transitions can take place at finite energies, but when a gap opens up, interband transitions become allowed, even at $\Gamma{ }^{23}$

Although the computed maps in the top row of Fig. 4 are shown unbroadened to highlight spectral features, the theoretical spectra shown in the bottom panels of Fig. 4 [blue (black) lines] have been smoothed to mimic the broadening

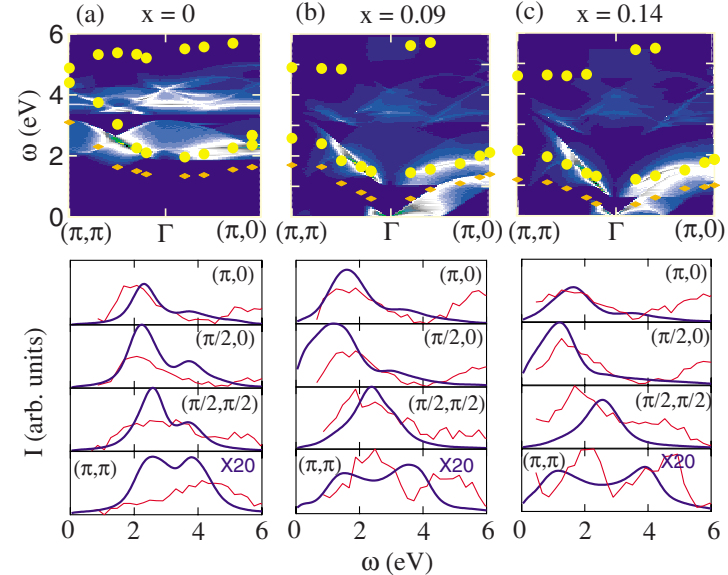

FIG. 4. (Color online) Top row: Doping evolution of RIXS spectra obtained within a three-band Hubbard model as a function of the energy transfer $\omega$ at momenta $\mathbf{q}$ along $\Gamma$ to $(\pi, \pi)$ and $(\pi, 0)$ directions for three different dopings $x$. The positions in $\omega-\mathbf{q}$ space of various experimentally observed spectral peaks (filled circles) and the leading edge of the low-energy feature (filled diamonds) are superposed. The computed spectra have not been broadened to reflect the experimental resolution in order to highlight spectral features. Bottom row: Comparison of theoretical [blue (black) lines] and experimental [red (gray) lines] spectra. The three columns refer to the three indicated doping levels $x$. Each column includes four different $q$ values as shown; the experimental spectra are at the closest $q$ value measured. The theoretical spectra have been smoothed to reflect experimental broadening (Ref. 22).

of experimental lineshapes. ${ }^{22}$ The relative intensities of the computed peaks are seen to be in agreement with our experimental results in lower energies ${ }^{24}$ except in the momentum region near the $(\pi, \pi)$ point, where both the experimental and the theoretical intensities are weak, but the computed cross section is smaller. A similar behavior is seen in the $\mathrm{La}_{2-x} \mathrm{Sr}_{x} \mathrm{CuO}_{4}$ series. ${ }^{9}$ Excitonic effects associated with longer range Coulomb coupling (intersite $V$ ) beyond that included in our model computations could enhance and redistribute the spectral weight near zone boundaries. ${ }^{25}$ The lowenergy, gap-edge dispersion relations ( $E$ vs $q)$ are in agreement as seen from the top rows of Figs. 4(a)-4(c).$^{23}$

In Fig. 5(b), we plot the doping dependence of the leading-edge gap (edge, filled squares) and peak position (peak, white diamonds) of the lowest branch at $\Gamma$. [Since quasielastic scattering makes it difficult to obtain experimental data right at $\Gamma$, these values are found by extrapolation from nearby points, as shown in Fig. 5(c).] Within the experimental uncertainties, the gap closes in the vicinity of the $\Gamma$ point as one approaches the critical doping regime in the phase diagram. For comparison, in Fig. 5(b), we also show $\Delta_{m}=U m_{d}$, which is the antiferromagnetic-correlation gap $^{7,10}$ based on our present calculation (filled circles) and the corresponding single particle spectrum $(\mathrm{SPS})^{10,26}$ results (triangles), which are seen to be in agreement. In general, our analysis suggests that the anisotropic softening involving a large energy scale observed near the Mott insulating state requires going beyond the one-band model.

In conclusion, we have utilized the unique momentum resolution of $\mathrm{x}$-ray scattering to map the evolution of 

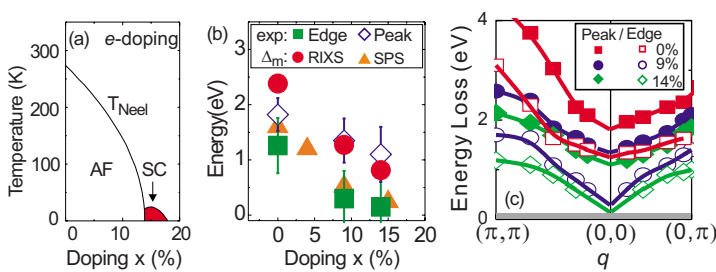

FIG. 5. (Color online) (a) Schematic temperature-doping (T-x) phase diagram of NCCO. (b) The leading-edge gap (filled squares) and peak positions (white diamonds) of the lowest-energy excitation branch in RIXS data at the $\Gamma$ point are compared to antiferromagnetic-correlation gap $\Delta_{m}$ from the present calculation (filled circles) and from SPS (triangles) (Ref. 10) as a function of doping. (c) The excitation modes (branches) are fitted and extrapolated to the $\Gamma$ point $(0,0)$ based on the branch curvatures to extract the data points presented in (b). The peak positions correspond to markings in Figs. 2(a)-2(c) and the leading edges are extracted from data presented in Fig. 3 .

particle-hole excitations in the electron-doped cuprate from its parent Mott state $(x=0)$ to the doping onset for supercon- ductivity. We observe a nearly degenerate charge excitation mode near the $(\pi, \pi)$ point around $5 \mathrm{eV}$ in the Mott insulator, which splits on doping away from half-filling, with its lowerenergy branch anisotropically softening in approaching the AFM/SC critical doping. In contrast, the response near the $(\pi, 0)$ wave vector exhibits damping with relatively little softening. Our results indicate the importance of multibandcorrelation structures in the collective charge dynamics of Mott insulators when electrons are added into the parent Mott state, which is in clear contrast to what is observed in hole-doped Mott insulators.

We gratefully acknowledge P. W. Anderson, N. P. Ong, and T. Tohyama for discussions and T. Gog and D. Casa for beamline support. This work is primarily supported by DOE/ BES Grant No. DE-FG-02-05ER46200. The work at NEU is supported by the U.S. Department of Energy Contract Nos. DE-FG02-07ER46352 and No. DE-AC03-76SF00098, and benefitted from the allocation of supercomputer time at NERSC and Northeastern University's Advanced Scientific Computation Center. The use of APS is supported by DOE W-31-10-Eng-38.
${ }^{1}$ M. Imada, A. Fujimori, and Y. Tokura, Rev. Mod. Phys. 70, 1039 (1998).

${ }^{2}$ M. A. Kastner, R. J. Birgeneau, G. Shirane, and Y. Endoh, Rev. Mod. Phys. 70, 897 (1998).

${ }^{3}$ G. Blumberg, M. Kang, M. V. Klein, K. Kadowaki, and C. Kendziora, Science 278, 1427 (1997).

${ }^{4}$ M. Z. Hasan, E. D. Isaacs, Z.-X. Shen, L. L. Miller, K. Tsutsui, T. Tohyama, and S. Maekawa, Science 288, 1811 (2000); Y. J. Kim, J. P. Hill, C. A. Burns, S. Wakimoto, R. J. Birgeneau, D. Casa, T. Gog, and C. T. Venkataraman, Phys. Rev. Lett. 89, 177003 (2002).

${ }^{5}$ J. P. Hill, C.-C. Kao, W. A. L. Caliebe, M. Matsubara, A. Kotani, J. L. Peng, and R. L. Greene, Phys. Rev. Lett. 80, 4967 (1998); P. Abbamonte, C. A. Burns, E. D. Isaacs, P. M. Platzman, L. L. Miller, S. W. Cheong, and M. V. Klein, ibid. 83, 860 (1999).

${ }^{6}$ K. Tsutsui, T. Tohyama, and S. Maekawa, Phys. Rev. Lett. 83, 3705 (1999); T. P. Devereaux, G. E. D. McCormack, and J. K. Freericks, ibid. 90, 067402 (2003); T. Nomura and J.-i. Igarashi, Phys. Rev. B 71, 035110 (2005); J. V. D. Brink and M. V. Veenendaal, Europhys. Lett. 73, 121 (2006).

${ }^{7}$ R. S. Markiewicz and A. Bansil, Phys. Rev. Lett. 96, 107005 (2006).

${ }^{8}$ M. Z. Hasan, P. A. Montano, E. D. Isaacs, Z.-X. Shen, H. Eisaki, S. K. Sinha, Z. Islam, N. Motoyama, and S. Uchida, Phys. Rev. Lett. 88, 177403 (2002).

${ }^{9}$ Y.-J. Kim, J. P. Hill, S. Komiya, Y. Ando, D. Casa, T. Gog, and C. T. Venkataraman, Phys. Rev. B 70, 094524 (2004); K. Ishii, K. Tsutsui, Y. Endoh, T. Tohyama, K. Kuzushita, T. Inami, K. Ohwada, S. Maekawa, T. Masui, S. Tajima, Y. Murakami, and J. Mizuki, Phys. Rev. Lett. 94, 187002 (2005).

${ }^{10}$ C. Kusko, R. S. Markiewicz, M. Lindroos, and A. Bansil, Phys. Rev. B 66, 140513(R) (2002).

${ }^{11}$ H. Kusunose and T. M. Rice, Phys. Rev. Lett. 91, 186407 (2003).

${ }^{12}$ D. Sénéchal and A.-M. S. Tremblay, Phys. Rev. Lett. 92, 126401 (2004).
${ }^{13}$ B. Kyung, V. Hankevych, A.-M. Daré, and A.-M. S. Tremblay, Phys. Rev. Lett. 93, 147004 (2004).

${ }^{14}$ Q. Yuan, F. Yuan, and C. S. Ting, Phys. Rev. B 72, 054504 (2005).

${ }^{15}$ K. Ishii, K. Tsutsui, Y. Endoh, T. Tohyama, S. Maekawa, M. Hoesch, K. Kuzushita, M. Tsubota, T. Inami, J. Mizuki, Y. Murakami, and K. Yamada, Phys. Rev. Lett. 94, 207003 (2005).

${ }^{16}$ N. P. Armitage et al., Phys. Rev. Lett. 88, 257001 (2002).

${ }^{17}$ P. K. Mang, O. P. Vajk, A. Arvanitaki, J. W. Lynn, and M. Greven, Phys. Rev. Lett. 93, 027002 (2004).

${ }^{18}$ R. S. Markiewicz, Phys. Rev. B 70, 174518 (2004).

${ }^{19}$ A. Zimmers, L. Shi, D. C. Schmadel, W. M. Fisher, R. L. Greene, H. D. Drew, M. Houseknecht, G. Acbas, M.-H. Kim, M.-H. Yang, J. Cerne, J. Lin, and A. Millis, Phys. Rev. B 76, 064515 (2007).

${ }^{20}$ J. W. Seo, K. Yang, D. W. Lee, Y. S. Roh, J. H. Kim, H. Eisaki, H. Ishii, I. Jarrige, Y. Q. Cai, D. L. Feng, and C. Kim, Phys. Rev. B 73, 161104(R) (2006).

${ }^{21}$ Y. Tokura, S. Koshihara, T. Arima, H. Takagi, S. Ishibashi, T. Ido, and S. Uchida, Phys. Rev. B 41, 11657 (1990).

${ }^{22}$ The spectra were first smoothed with a Gaussian of $0.37 \mathrm{eV}$ width to account for the resolution. The spectra were further smoothed via folding with a Lorentzian of $0.30 \mathrm{eV}$ width to mimic what appears to be an intrinsic linewidth.

${ }^{23}$ The branch near $4 \mathrm{eV}$ is weak in the experimental data. Since this branch has a different symmetry than the $2 \mathrm{eV}$ branch, it resonates at a different photon energy (near $8.894 \mathrm{eV}$ ), as evident from the raw data profile in Fig. 1(f).

${ }^{24}$ The experimental spectra for energy transfers beyond about $6 \mathrm{eV}$ contain significant contributions from bands beyond the threeband model we considered for this work.

${ }^{25}$ K. Penc and W. Stephan, Phys. Rev. B 62, 12707 (2000).

${ }^{26}$ In the one-band model, $U$ has a different definition and the magnetic gap is $\Delta_{m}=2 U m_{d}$. Also, at $x=0$, ARPES does not see the upper band giving a lower limit for $\Delta_{m}$. 